\title{
Migdal-Eliashberg theory of multi-band high-temperature superconductivity in field-effect-doped hydrogenated (111) diamond
}

\author{
Davide Romanin, ${ }^{1, *}$ Giovanni A. Ummarino, ${ }^{1,2}$ and Erik Piatti ${ }^{1, \dagger}$ \\ ${ }^{1}$ Department of Applied Science and Technology, Politecnico di Torino, 10129 Torino, Italy \\ ${ }^{2}$ National Research Nuclear University MEPhI (Moscow Engineering \\ Physics Institute), Kashira Hwy 31, Moskva 115409, Russia
}

\begin{abstract}
We perform single- and multi-band Migdal-Eliashberg (ME) calculations with parameters exctracted from density functional theory (DFT) simulations to study superconductivity in the electricfield-induced 2-dimensional hole gas at the hydrogenated (111) diamond surface. We show that according to the Eliashberg theory it is possible to induce a high- $T_{c}$ superconducting phase when the system is field-effect doped to a surface hole concentration of $6 \times 10^{14} \mathrm{~cm}^{-2}$, where the Fermi level crosses three valence bands. Starting from the band-resolved electron-phonon spectral functions $\alpha^{2} F_{j j^{\prime}}(\omega)$ computed ab initio, we iteratively solve the self-consistent isotropic Migdal-Eliashberg equations, in both the single-band and the multi-band formulations, in the approximation of a constant density of states at the Fermi level. In the single-band formulation, we find $\mathrm{T}_{\mathrm{c}} \approx 40 \mathrm{~K}$, which is enhanced between $4 \%$ and $8 \%$ when the multi-band nature of the system is taken into account. We also compute the multi-band-sensistive quasiparticle density of states to act as a guideline for future experimental works.
\end{abstract}

Keywords: diamond, density functional theory, ionic gating, electron-phonon interaction, Eliashberg theory, multi-band superconductivity

\section{INTRODUCTION}

Superconductivity (SC) in diamond was first reported in 2004 by E. A. Ekimov et al. ${ }^{1}$ by boron (B) substitution of carbon (C) atoms, leading to a hole-type conductivity and to a transition temperature $T_{\mathrm{c}}=4 \mathrm{~K}^{1,2}$ at a dopant concentration $n_{\mathrm{B}} \approx 4-5 \cdot 10^{21} \mathrm{~cm}^{-3}$. It was then confirmed theoretically that the B-doped bulk diamond is a conventional phonon-mediated superconductor, and that by increasing the $\mathrm{B}$ concentration it would be possible to enhance $T_{\mathrm{c}}{ }^{3-8}$. From the experimental point of view, however, the solubility limit ${ }^{9,10}$ of B in the closelypacked diamond structure prevents increasing the doping further, thus limiting the highest achievable $T_{\mathrm{c}}$. An alternative route to induce hole-type conductivity in diamond is by hydrogen $(\mathrm{H})$ termination followed by exposure to electron-accepting molecules such as air moisture ${ }^{11-13}$, leading to the formation of a surface-bound high-mobility two-dimensional hole gas whose properties have been extensively investigated ${ }^{11-18}$. Furthermore, the carrier density in hydrogenated diamond can be increased by means of electric field-effect doping: The configuration is that of a field-effect transistor (FET), i.e. an asymmetric capacitor where the material under study is separated from a metallic gate by a suitable dielectric. The application of a finite voltage between the gate and the sample $\left(V_{\mathrm{G}}\right)$ allows to accumulate charges-carriers in the first few layers of the sample (whose sign depends on that of $V_{\mathrm{G}}$ ) which screen the resulting applied electric field [Fig. 1(a)]. While a solid dielectric allows for an effective doping of the order of $\sim 10^{12}-10^{13} \mathrm{~cm}^{-2}$, the use of an ionic liquid or a polymer-electrolyte solution can increase it to $\sim 10^{14}-10^{15} \mathrm{~cm}^{-219-29}$. In the specific case of diamond, electrolyte-based gating can routinely tune hole dopings up to $\sim 7 \times 10^{13} \mathrm{~cm}^{-230-36}$, and a proper engineering of the surface properties can extend the range at least to few units in $10^{14} \mathrm{~cm}^{-230,36,37}$.

Previous density functional theory (DFT) simulations $^{30,38-40}$ showed that it is possible to induce a SC phase transition in hydrogenated diamond surfaces by hole-doping in the FET configuration. In these studies, the critical temperature $T_{\mathrm{c}}$ was estimated using the McMillan/Allen-Dynes ${ }^{41,42}$ formula. In particular, in Refs. 38 and 39 the authors studied the hydrogenated diamond (110) surface, and predicted a SC phase transition at $T_{\mathrm{c}} \sim 1 \mathrm{~K}$. In Ref. 40, on the other hand, it was shown that the hydrogenated diamond (111) surface develops multi-band $\mathrm{SC}$ with a much higher $T_{\mathrm{c}} \sim 30 \mathrm{~K}$. However, the McMillan/Allen-Dynes formula only gives qualitative results: A more accurate estimate of $T_{\mathrm{c}}$ necessitates solving the Migdal-Eliashberg (ME) equations instead $^{43,44}$.

In this work - starting from the results of Ref. 40 - we study the electric-field-induced high- $T_{\mathrm{c}} \mathrm{SC}$ in the hydrogenated diamond (111) surface via the full self-consistent solution of the ME equations. In particular, we focus on the role of the multi-band Fermi surface on the SC pairing and $T_{\mathrm{c}}$. In Sec. II we summarize the main DFT results obtained by Ref. 40 at the field-induced hole doping of $n_{\mathrm{dop}}=6 \times 10^{14} \mathrm{~cm}^{-2}$, and introduce the multi-band Eliashberg equations on the imaginary axis, discussing also the Ansatz made for the effective electron-electron (e-e) interaction. In Sec. III we move to the solution of the isotropic Eliashberg equations, first in the singleband approximation and then including the multi-band nature of the system (always in the approximation of a constant density of states at the Fermi level). We also determine the quasiparticle density of states from the analytic continuation of the SC gaps to the real axis. 


\section{MODEL AND METHODS}

\section{A. DFT computation of the electron-phonon matrix elements}

In Ref. 40 it was shown that a SC phase transition can be induced in the hydrogenated diamond (111) surface via field-effect doping at a hole concentration of $n_{\text {dop }}=6 \times 10^{14} \mathrm{~cm}^{-2}$. This was done by performing DFT calculations in the proper field-effect geometry with Quantum ESPRESSO ${ }^{45-47}$ as described in Ref. 47 in the case of graphene: This is a very versatile approach, which has been employed to reliably calculate the properties of many different materials from first principles, including zirconium nitride chloride ${ }^{48}$, arsenene and phosphorene ${ }^{49}$, transition-metal dichalcogenides $^{49-53}$, niobium nitride ${ }^{54}$, and silicon ${ }^{55}$. For fieldeffect doped (111) diamond, all the computational details can be found in Ref. 40. As shown in Fig. 1(a), we have considered a slab made of $14 \mathrm{C}$ layers, terminated on both side by $\mathrm{H}$ atoms in order to saturate dangling bonds and avoid the surface reconstruction ${ }^{30,40}$, for a total of 16 atoms in the unit cell. The presence of the $\mathrm{H}$ atoms on both side of the slab is also necessary to have a symmetric system as prescribed by the field-effect geometry implementation of Ref. ${ }^{47}$, where the metallic gate is represented by a planar uniform distribution of point charges separated from the slab by a potential barrier to prevent charge spilling. Approximatively $30 \AA$ of vacuum has also been inserted between different repeated images to avoid interferences between them due to the periodic boundary conditions.

At $n_{\text {dop }}=6 \times 10^{14} \mathrm{~cm}^{-2}$, the field-induced twodimensional hole gas (2DHG) is multi-band in nature, since at the Fermi level (we set $E_{\mathrm{F}}=0$ ) three holonic bands are crossed. As a consequence, the Fermi surface is composed of three non-crossing concentric hole pockets [Fig. 1(b)] centered around $\boldsymbol{\Gamma}$ (i.e. the center of the Brillouin zone), which appear to be isotropic in $\mathbf{k}$-space (where k-points denote the electron momenta). Tab. I summarizes the total electronic density of states $N_{\sigma}(0)$ and the DOS for each band $N_{\sigma, j}(0)$ at the Fermi level, the corresponding band-averaged and band-resolved Fermi wavevectors, $\left\langle k_{\mathrm{F}}\right\rangle$ and $\left\langle k_{\mathrm{F}}\right\rangle_{j}$, and the band-averaged and band-resolved Fermi velocities, $\left\langle v_{\mathrm{F}}\right\rangle$ and $\left\langle v_{\mathrm{F}}\right\rangle_{j}$. Here, $j=1,2,3$ is the band index as labelled in Fig. 1(b). We also stress that, since the field-induced 2DHG is strongly confined to the surface ${ }^{40}$, the DOS is step-like: The assumption of constant DOS at the Fermi level is thus satisfied. The band-resolved Fermi velocities are calculated by averaging over each Fermi surface as:

$$
\left\langle v_{\mathrm{F}}\right\rangle_{j}=\left.\frac{1}{2 \pi} \int_{0}^{2 \pi} d \theta \frac{1}{\hbar} \frac{\partial \epsilon_{j, \mathbf{k}}}{\partial k}\right|_{\epsilon_{j, \mathbf{k}}=E_{\mathrm{F}}}
$$

where $\hbar$ is the reduced Planck constant, $\epsilon_{j, \mathbf{k}}$ is the energy dispersion of the $j$-th band in $\mathbf{k}$-space, $k=|\mathbf{k}|$ and $\theta$ is the in-plane angular coordinate in $\mathbf{k}$-space. The bandaveraged Fermi velocity is the weighted average of the

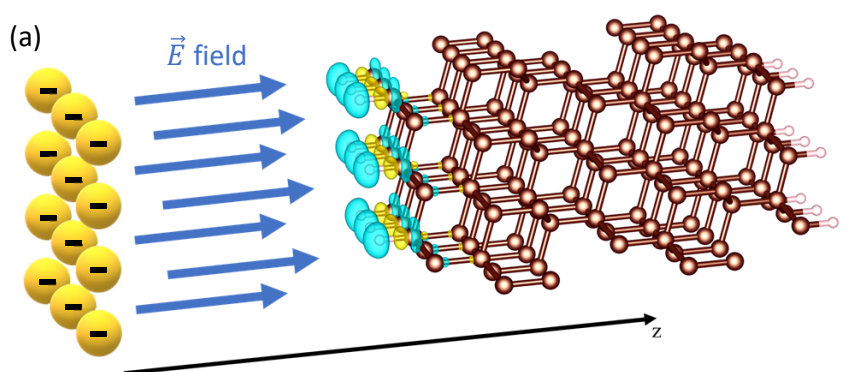

(b) $\frac{2 \pi}{a}$
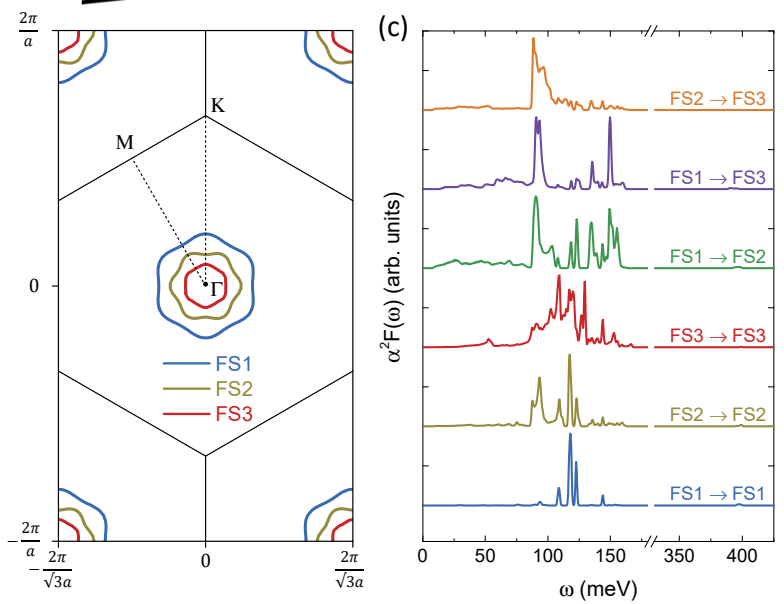

FIG. 1. (a) Schematic view of the hydrogenated diamond (111) surface in the FET configuration. Brown spheres are C atoms, while pink spheres are $\mathrm{H}$ atoms. Light-blue (yellow) regions represents the induced hole (electron) charge densities on the surface. Blue arrows denote the electric field (E) normal to the surface; (b) Fermi surface of the hydrogenated diamond (111) surface doped with $n_{\text {dop }}=6 \times 10^{14} \mathrm{~cm}^{-2}$. FS1, FS2 and FS3 label the three different Fermi sheets; (c) Interand intra-band Eliashberg functions, normalized to 1 , of the hydrogenated diamond (111) surface doped at $n_{\text {dop }}=6 \times 10^{14}$ $\mathrm{cm}^{-2}$. The curves are in arbitrary units and vertically shifted for clarity.

band-resolved Fermi velocities by the corresponding DOS at the Fermi level:

$$
\left\langle v_{\mathrm{F}}\right\rangle=\frac{\sum_{j} N_{\sigma, j}(0)\left\langle v_{\mathrm{F}}\right\rangle_{j}}{\sum_{j} N_{\sigma, j}(0)}
$$

We then performed linear response computations on a coarse grid of phonon momenta (q-points) in the Brillouin zone in order to compute the electron-phonon (eph) matrix elements $g_{j \mathbf{k}, j^{\prime} \mathbf{k}^{\prime}}^{\nu}$ for the phonon mode $\nu$ and between electronic states $|j \mathbf{k}\rangle$ and $\left|j^{\prime} \mathbf{k}^{\prime}\right\rangle$ :

$$
g_{j \mathbf{k}, j^{\prime} \mathbf{k}^{\prime}}^{\nu}=\sum_{A \alpha} \frac{e_{\mathbf{k}^{\prime}-\mathbf{k} \nu}^{A \alpha}}{\sqrt{2 M_{A} \omega_{\mathbf{k}^{\prime}-\mathbf{k} \nu}}}\left\langle\mathbf{k} j\left|\frac{\delta v_{\mathrm{SCF}}}{\delta u_{A \alpha}^{\mathbf{k}^{\prime}-\mathbf{k}}}\right| j^{\prime} \mathbf{k}^{\prime}\right\rangle
$$

where $j$ is the band index, $\mathbf{k}^{\prime}=\mathbf{k}+\mathbf{q}$, and $v_{\mathrm{SCF}}=$ $V_{\mathrm{KS}} e^{-i\left(\mathbf{k}^{\prime}-\mathbf{k}\right) \cdot \mathbf{r}}$ is the periodic part of the Kohn-Sham potential. $A$ labels atoms in the unit cell, $M_{A}$ is the mass of the $A$-th atom and $\alpha=\mathrm{x}, \mathrm{y}, \mathrm{z}$ (i.e. the cartesian coordinates). $u_{A \alpha}^{\mathbf{k}-\mathbf{k}^{\prime}}$ is the Fourier-transformed displacement 
of the $A$-th atom along $\alpha$ and the cartesian component of the normalized phonon eigenvector on the unit cell is $e_{\mathbf{k}^{\prime}-\mathbf{k} \nu}^{A \alpha}$. Then, we exploited Wannier functions ${ }^{56}$ in order to interpolate the e-ph matrix elements over the whole Brillouin zone, as described in Ref. 57. By doing so it was possible to compute the band-resolved Eliashberg spectral function $\alpha^{2} F_{j j^{\prime}}(\omega)$ [Fig. 1(c)] which gives the frequency-dependent e-ph interaction ${ }^{58}$ :

$$
\begin{aligned}
\alpha^{2} F_{j j^{\prime}}(\omega)= & \frac{1}{N_{j, \sigma}^{2}(0) N_{k} N_{q}} \sum_{\mathbf{k k}^{\prime}} \alpha^{2} F_{j \mathbf{k}, j^{\prime} \mathbf{k}^{\prime}}(\omega) \delta\left(\epsilon_{j \mathbf{k}}\right) \delta\left(\epsilon_{j^{\prime} \mathbf{k}^{\prime}}\right) \\
= & \frac{1}{N_{j, \sigma}(0) N_{k} N_{q}} \sum_{\mathbf{k}^{\prime} \nu} \delta\left(\omega-\omega_{\mathbf{q} \nu}\right) . \\
& \cdot \sum_{\mathbf{k}}\left|g_{j \mathbf{k} j^{\prime} \mathbf{k}^{\prime}}^{\nu}\right|^{2} \delta\left(\epsilon_{j \mathbf{k}}\right) \delta\left(\epsilon_{j^{\prime} \mathbf{k}^{\prime}}\right)
\end{aligned}
$$

where $N_{k}\left(N_{q}\right)$ is the total number of $\mathbf{k}$-points (q-points) used in the sum. The Dirac-delta $\delta\left(\epsilon_{j \mathbf{k}}\right)$ and $\delta\left(\epsilon_{j^{\prime} \mathbf{k}^{\prime}}\right)$ limit the scattering events to the Fermi surface. We also calculate the band-resolved e-ph coupling constant $\lambda_{j j^{\prime}}$, i.e. the average strength of the e-ph interaction involving scattering events between the $j$-th and $j^{\prime}$-th bands:

$$
\lambda_{j j^{\prime}}=2 \int d \omega \frac{\alpha^{2} F_{j j^{\prime}}(\omega)}{\omega}
$$

such that the total e-ph coupling constant, $\lambda$, is found as the weighted average of the band-resolved e-ph couplings, $\lambda_{j j^{\prime}}$, by the corresponding band-resolved DOS at the Fermi level, $N_{\sigma, j}(0)$ :

$$
\lambda=\frac{\sum_{j j^{\prime}} N_{\sigma, j}(0) \lambda_{j j^{\prime}}}{\sum_{j} N_{\sigma, j}(0)}
$$

In our case we found that the total e-ph coupling constant is $\lambda=0.81$, while its decomposition on the electronic bands $\left(j, j^{\prime}=1,2,3\right.$ for the present system) gives a $3 \times 3$

\begin{tabular}{cccc}
\hline Global & $N_{\sigma}(0)$ & $\left\langle k_{\mathrm{F}}\right\rangle$ & $\left\langle v_{\mathrm{F}}\right\rangle$ \\
\hline Band-resolved & 0.3936 & 3.62 & 3.58 \\
\hline Band 1 & $N_{\sigma, j}(0)$ & $\left\langle k_{\mathrm{F}}\right\rangle_{j}$ & $\left\langle v_{\mathrm{F}}\right\rangle_{j}$ \\
Band 2 & 0.1783 & 4.81 & 3.71 \\
Band 3 & 0.1088 & 3.26 & 4.28 \\
\hline & 0.1059 & 2.02 & 2.65 \\
\hline & $\mathrm{eV}^{-1} \mathrm{spin}^{-1}(16 \text { atoms cell })^{-1}$ & $\AA^{-1}$ & $10^{5} \mathrm{~m} / \mathrm{s}$ \\
\hline
\end{tabular}

TABLE I. Main bandstructure parameters at the Fermi level $\left(E_{F}=0\right)$ for the field-effect-doped hydrogenated diamond (111) surface at a doping level of $n_{\text {dop }}=6 \times$ $10^{14} \mathrm{~cm}^{-2}$ : Total DOS $N_{\sigma}(0)$, band-resolved DOS $N_{\sigma, j}(0)$, band-averaged Fermi wavevector $\left\langle k_{\mathrm{F}}\right\rangle$, band-resolved Fermi wavevector $\left\langle k_{\mathrm{F}}\right\rangle_{j}$, band-averaged Fermi velocity $\left\langle v_{\mathrm{F}}\right\rangle$, and band-resolved Fermi velocity $\left\langle v_{\mathrm{F}}\right\rangle_{j}$. The indexes $j=1,2,3$ are labelled in Fig. 1(b). matrix:

$$
\lambda_{j j^{\prime}}=\left(\begin{array}{lll}
0.4629 & 0.2425 & 0.1984 \\
0.3973 & 0.2167 & 0.1806 \\
0.3340 & 0.1856 & 0.1484
\end{array}\right)
$$

The SC critical temperature $T_{\mathrm{c}}$ was estimated using the McMillan/Allen-Dynes ${ }^{41,42}$ formula:

$$
T_{\mathrm{c}}=\frac{\omega_{\log }}{1.2} \exp \left\{-\frac{1.04(1+\lambda)}{\lambda-\mu^{*}(1+0.62 \lambda)}\right\}
$$

where $\omega_{\log }$ is the logarithmic-averaged phonon frequency, computed to be $\omega_{\log }=83 \mathrm{meV}$, and $\mu^{*}$ is the MorelAnderson pseudopotential ${ }^{59}$, i.e. a measure of the effective e-e interaction. Since $\mu^{*}$ is an ad-hoc parameter, we assumed that its value falls within the same range as that of $\mathrm{SC}$ boron-doped bulk diamond ${ }^{3}$, that is $\mu^{*} \in[0.13 ; 0.14]$. The field-doped hydrogenated (111) diamond surface is then predicted to undergo a SC phase transition in the range of $T_{\mathrm{c}} \in[29.6 ; 34.9] \mathrm{K}$. In order to highlight the difference between inter- and intra-band couplings, we consider the symmetrized electron-phonon coupling constant matrix $\tilde{\lambda}_{j j^{\prime}}=N_{\sigma, j}(0) \lambda_{j j^{\prime}} / N_{\sigma}(0)$ :

$$
\lambda_{j j^{\prime}}=\left(\begin{array}{lll}
0.21 & 0.11 & 0.09 \\
0.11 & 0.06 & 0.05 \\
0.09 & 0.05 & 0.04
\end{array}\right)
$$

where the diagonal elements describe the intra-band couplings, while the off-diagonal elements describe the interband ones. From Eq. 9 we can see that the most relevant processes are those concerning the intra-band scattering on the first Fermi surface (FS1 $\leftrightarrow$ FS1) and the inter-band scattering of the second (FS2 $\leftrightarrow \mathrm{FS} 1$ ) and third Fermi surfaces $(\mathrm{FS} 3 \leftrightarrow \mathrm{FS} 1)$ with the first one.

\section{B. Imaginary-axis Migdal-Eliashberg equations}

The multi-band fully-anisotropic k-resolved Eliashberg equations on the imaginary axis ${ }^{43,44,60-63}$ consist in a set of $3 j$ non-linear coupled equations which have to be solved self-consistently:

$$
\begin{aligned}
Z_{j \mathbf{k}}\left(i \omega_{n}\right)= & 1+\frac{1}{\beta N_{\sigma}(0) \omega_{n}} \sum_{\mathbf{k}^{\prime} n^{\prime} j^{\prime}} \frac{\omega_{n^{\prime}} Z_{j^{\prime} \mathbf{k}^{\prime}}\left(i \omega_{n^{\prime}}\right)}{\Xi_{j^{\prime} \mathbf{k}^{\prime}}^{2}\left(i \omega_{n^{\prime}}\right)} . \\
& \cdot \lambda_{j \mathbf{k}, j^{\prime} \mathbf{k}^{\prime}}\left(\omega_{n}-\omega_{n^{\prime}}\right) \\
\phi_{j \mathbf{k}}\left(i \omega_{n}\right)= & \frac{1}{\beta N_{\sigma}(0)} \sum_{\mathbf{k}^{\prime} n^{\prime} j^{\prime}} \frac{\phi_{j^{\prime} \mathbf{k}^{\prime}}\left(i \omega_{n^{\prime}}\right)}{\Xi_{j^{\prime} \mathbf{k}^{\prime}}^{2}\left(i \omega_{n^{\prime}}\right)} \cdot \\
& \left.\cdot\left[\lambda_{j \mathbf{k}, j^{\prime} \mathbf{k}^{\prime}}\left(\omega_{n}-\omega_{n^{\prime}}\right)-\mu_{j j^{\prime}}^{*} \theta\left(\omega-\left|\omega_{c}\right|\right)\right] 10 \mathrm{~b}\right) \\
\chi_{j \mathbf{k}}\left(i \omega_{n}\right)= & -\frac{1}{\beta N_{\sigma}(0)} \sum_{\mathbf{k}^{\prime} n^{\prime} j^{\prime}} \frac{\epsilon_{j^{\prime} \mathbf{k}^{\prime}}+\chi_{j^{\prime} \mathbf{k}^{\prime}}\left(i \omega_{n^{\prime}}\right)}{\Xi_{j^{\prime} \mathbf{k}^{\prime}}^{2}\left(i \omega_{n^{\prime}}\right)} . \\
& \cdot \lambda_{j \mathbf{k}, j^{\prime} \mathbf{k}^{\prime}}\left(\omega_{n}-\omega_{n^{\prime}}\right)
\end{aligned}
$$


where $\omega_{n}=(2 n+1) / \beta$ are the Matsubara's frequencies for fermions (with $n$ an integer number), $\beta=1 / T$ is the inverse temperature (we set the Boltzmann constant $\left.k_{B}=1\right), \mu_{j j^{\prime}}^{*}$ is the effective Coulomb interaction between electrons on the $j$-th band and those on the $j^{\prime}$-th band and the energies are measured from the Fermi level $\left(E_{F}=0\right) . \Xi_{j}^{2}\left(\mathbf{k}, i \omega_{n}\right)$ is defined as:

$$
\begin{aligned}
\Xi_{j \mathbf{k}}^{2}\left(i \omega_{n}\right) & =\omega_{n}^{2} Z_{j \mathbf{k}}^{2}\left(i \omega_{n}\right)+ \\
& +\left(\epsilon_{j \mathbf{k}}+\chi_{j \mathbf{k}}\left(i \omega_{n}\right)\right)^{2}+\phi_{j \mathbf{k}}^{2}\left(i \omega_{n}\right)
\end{aligned}
$$

In order to avoid the divergence of the sums over $n$ in Eq. 10, the number of Matsubara's frequency is limited by a cut-off energy $\omega_{c}$ which goes typically from $\omega_{c} \sim$ $4 \omega_{\max }$ to $\omega_{c} \sim 10 \omega_{\max }$ (with $\omega_{\max }$ the maximum phonon frequency). In our case we choose $\omega_{c}=1290 \mathrm{meV}$. The e-ph coupling constant is then given by:

$$
\begin{aligned}
\lambda_{j \mathbf{k}, j^{\prime} \mathbf{k}^{\prime}}\left(\omega_{n}-\omega_{n^{\prime}}\right)= & \int_{0}^{\omega_{\max }} d \omega \frac{2 \omega}{\left(\omega_{n}-\omega_{n^{\prime}}\right)^{2}+\omega^{2}} . \\
& \cdot \alpha^{2} F_{j \mathbf{k}, j^{\prime} \mathbf{k}^{\prime}}(\omega)
\end{aligned}
$$

Moreover, in order to preserve the number of electrons in the system, the Fermi level must be re-computed selfconsistently according to:

$$
N=1-\frac{2}{\beta} \sum_{\mathbf{k} n j} \frac{\epsilon_{j \mathbf{k}}+\chi_{j \mathbf{k}}\left(i \omega_{n}\right)}{\Xi_{j \mathbf{k}}^{2}\left(i \omega_{n}\right)}
$$

Therefore, in the present work we would need to solve self-consistently a set of 10 equations for the fully anisotropic system. Finally, the order parameter (i.e. the $\mathrm{SC}$ energy gap) for each band is defined as:

$$
\Delta_{j \mathbf{k}}\left(i \omega_{n}\right)=\frac{\phi_{j \mathbf{k}}\left(i \omega_{n}\right)}{Z_{j \mathbf{k}}\left(i \omega_{n}\right)}
$$

The order parameters have to be computed for different values of the temperature $T$, i.e. the set of equations Eq. 10 have to be solved for each value of $T$. The SC critical temperature $T_{\mathrm{c}}$ is found when all of the $j$ energy gaps are zero, i.e. when $\Delta_{j \mathbf{k}}\left(i \omega_{n} ; T_{\mathrm{c}}\right)=0$.

In order to simplify the computational cost, the DOS is assumed to be constant in an energy region of $2 \hbar \omega_{\max }$ around the Fermi energy. Therefore we can restrict ourselves only to a small energy range around $E_{\mathrm{F}}$ : in such a way Eq. 10c is exactly zero and Eq. 13 can be neglected, reducing the problem to the self-consistent solution of only 6 non-linear coupled equations. Moreover, for smooth and well-behaved (i.e non-crossing and isotropic) Fermi surfaces, we can pass from a k-resolved picture to an energy description by averaging Eq. 10a and Eq. 10b over the the Fermi surface. In this case we are left to

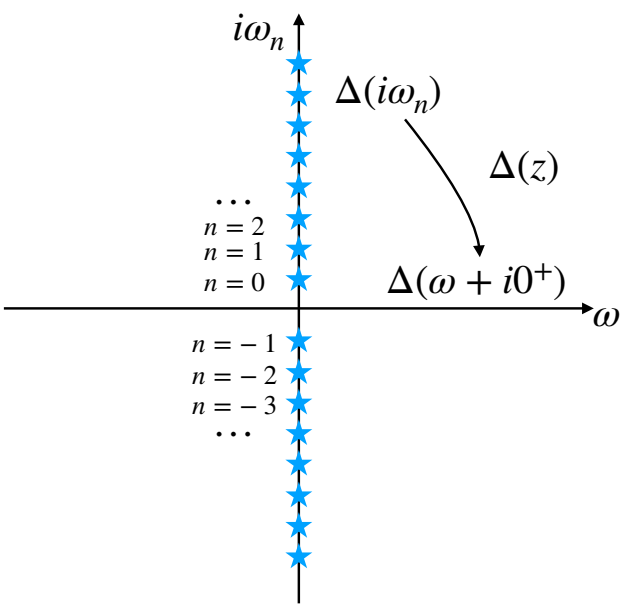

FIG. 2. Analytic continuation of the superconducting energy gap from the imaginary axis $\left(\Delta\left(i \omega_{n}\right)\right)$ to the real axis $\left(\Delta\left(\omega+i 0^{+}\right)\right)$. Blue stars denotes the values of $\Delta\left(i \omega_{n}\right)$ for $n=\ldots,-2,-1,0,1,2, \ldots$.

solve the isotropic ME equations:

$$
\begin{aligned}
& Z_{j}\left(i \omega_{n}\right)=1+\frac{\pi}{\beta \omega_{n}} \sum_{n^{\prime} j^{\prime}} \frac{\omega_{n^{\prime}}}{S_{j^{\prime}}\left(i \omega_{n^{\prime}}\right)} . \\
& \cdot \lambda_{j j^{\prime}}\left(i \omega_{n}, i \omega_{n^{\prime}}\right) \\
& Z_{j}\left(i \omega_{n}\right) \Delta_{j}\left(i \omega_{n}\right)=\frac{\pi}{\beta} \sum_{n^{\prime} j^{\prime}} \frac{\Delta_{j^{\prime}}\left(i \omega_{n^{\prime}}\right)}{S_{j^{\prime}}\left(i \omega_{n^{\prime}}\right)} . \\
& \cdot\left[\lambda_{j j^{\prime}}\left(i \omega_{n}, i \omega_{n^{\prime}}\right)-\mu_{j j^{\prime}}^{*} \theta\left(\omega-\omega_{c}\right)\right]
\end{aligned}
$$

Thanks to Padé approximants ${ }^{64}$ it is possible to analitically continue ${ }^{65,66}$ the SC gap (Eq. 14) from the imaginary axis to the real axis (Fig. 2). Once we have the frequency dependence of the order parameter along the real axis, it is possible to extract the quasiparticle DOS in the SC phase $N_{S, \sigma j}(\omega)$ through:

$$
\frac{N_{S, \sigma j}(\omega)}{N_{\sigma j}(0)}=\mathfrak{R e}\left[\frac{\omega}{\sqrt{\omega^{2}-\Delta_{j}^{2}(\omega)}}\right]
$$

Notice that in the single-band case the label $j$ is no longer needed and we simply have $\mu_{j j^{\prime}}^{*}=\mu^{*}$.

\section{Choice of the Coulomb pseudopotential $\mu_{j j^{\prime}}^{*}$}

The only free parameter in the ME theory is the effective Coulomb e-e repulsion, which is different ${ }^{61}$ from the Morel-Anderson pseudopotential of the McMillan/AllenDynes formula. Similarly to the e-ph coupling, the effective Coulomb pseudopotential is a scalar in the singleband model and a matrix in the multi-band model, in principle resulting in a large number of free parameters. In order to keep the problem tractable, in the latter case 
we must make an Ansatz on the form of the band-resolved $\mu_{j j^{\prime}}^{*}$. Its decomposition on the electronic bands is the following $3 \times 3$ matrix:

$$
\left(\begin{array}{ccc}
\mu_{11}^{*} & \mu_{12}^{*}=c \cdot \mu_{11}^{*} & \mu_{13}^{*}=c \cdot \mu_{11}^{*} \\
\mu_{21}^{*}=\frac{N_{\sigma, 1}(0)}{N_{\sigma, 2}(0)} \mu_{12}^{*} & \mu_{22}^{*}=\mu_{11}^{*} & \mu_{23}^{*}=c \cdot \mu_{11}^{*} \\
\mu_{31}^{*}=\frac{N_{\sigma, 1}(0)}{N_{\sigma, 3}(0)} \mu_{13}^{*} & \mu_{32}^{*}=\frac{N_{\sigma, 2}(0)}{N_{\sigma, 3}(0)} \mu_{23}^{*} & \mu_{33}^{*}=\mu_{11}^{*}
\end{array}\right)
$$

where $c$ is a number that we choose equal to either 0 , $1 / 2$ or 1 . For example, in the case of $\mathrm{MgB}_{2}$ (the most known two-band e-ph superconductor) this parameter is approximately $0<c<1 / 2^{67}$. We can then retrieve a total Coulomb pseudopotential $\mu_{\text {tot }}^{*}$ as:

$$
\mu_{\mathrm{tot}}^{*}=\frac{\sum_{j j^{\prime}} N_{\sigma, j}(0) \mu_{j j^{\prime}}^{*}}{\sum_{j} N_{\sigma, j}(0)}
$$

We estimate the value of $\mu_{\text {tot }}^{*}$ most appropriate for the ME formalism by first solving the single-band isotropic ME equations (Eq. 15) in the case of boron-doped bulk diamond, which is known to undergo a SC phase transition at $T_{\mathrm{c}} \approx 4 \mathrm{~K}$ at a boron concentration of $\sim 1.85 \%$. Using the Eliashberg spectral function computed in Ref. 3, the experimental value of $T_{\mathrm{c}}$ can be found by setting $\mu_{\mathrm{tot}}^{*}=\mu^{*}=0.17$. This value will be considered representative also for the hydrogenated diamond (111) surface. In the multi-band case, the values of $\mu_{j j^{\prime}}^{*}$ are determined by $\mu_{\text {tot }}^{*}=0.17$ via Eq. 17 and the chosen value of $c$, as summarized in Table II.

\begin{tabular}{ccccc}
\hline & Single-band & \multicolumn{3}{c}{ Multi-band } \\
\hline & & $c=0$ & $c=1 / 2$ & $c=1$ \\
\hline$\mu_{\text {tot }}^{*}$ & 0.17 & 0.17 & 0.17 & 0.17 \\
$\mu_{j j^{\prime}, j=j^{\prime}}^{*}$ & $/$ & 0.17 & 0.07785 & 0.05048 \\
$\mu_{j j^{\prime}, j<j^{\prime}}^{*}$ & $/$ & 0 & 0.038925 & 0.05048 \\
\hline
\end{tabular}

TABLE II. Coulomb pseudopotentials employed in the solution of the single-band and multi-band Migdal-Eliashberg equations. All values are obtained under the condition that the total pseudopotential reproduces the experimental $T_{\mathrm{c}}$ of boron-doped bulk diamond. The indexes $j=1,2,3$ are labelled in Fig. 1(b).

\section{RESULTS}

\section{A. Isotropic single-band solution}

We start our analysis from a single-band isotropic description of our system, and compute the resulting dependence $T_{\mathrm{c}}$ for increasing values of $\mu^{*}$ (solid black line in Fig. 3). For a given value of $\mu^{*}$, this is done by computing the $T$-dependence of the $\mathrm{SC}$ order parameter on the imaginary axis $\Delta\left(i \omega_{n=0}\right)$, as shown in Fig. $4(\mathrm{a})$, and $T_{\mathrm{c}}$ is then found as the value of $T$ for which $\Delta\left(i \omega_{n}\right) \rightarrow 0$. For the representative value $\mu^{*}=0.17$, we find $T_{\mathrm{c}}=40.3 \mathrm{~K}$.
Note that this value is higher than the one obtained from the McMillan/Allen-Dynes formula $\left(T_{\mathrm{c}} \in[29.6 ; 34.9]\right.$, $\left.\mu^{*} \in[0.13 ; 0.14]\right)$, indicating that the latter significantly underestimates the value of $T_{\mathrm{c}}$ in our system. We determine the value of the SC order parameter in the $T \rightarrow 0$ limit, obtaining $\Delta\left(i \omega_{n=0} ; T=0\right)=6.46 \mathrm{meV}$, from which we can calculate the gap-to- $T_{\mathrm{c}}$ ratio:

$$
r=\frac{2 \Delta\left(i \omega_{n=0} ; T=0\right)}{k_{B} T_{c}}=3.72
$$

which is close to the BCS value of $r_{\mathrm{BCS}}=3.54$. Usually the gap-ratio (Eq. 18) requires the value of the superconducting gap computed on the real axis, however in our case the one computed on the imaginary-axis is not different (i.e. we are in a weak-coupling limit). We then perform the analytic continuation of the SC gap on the imaginary-axis at $T=T_{\mathrm{c}} / 10, \Delta\left(i \omega_{n} ; T=T_{\mathrm{c}} / 10\right)$, to the real-axis $[\Delta(\omega)$, Fig. $4(\mathrm{~b})]$. From this we finally compute the quasiparticle DOS [see inset to Fig. 4(a)], which shows the typical shape of an $s$-wave superconductor.

\section{B. Isotropic multi-band solution}

Having firmly established the description of our system in the single-band approximation, we now move to the more accurate multi-band description. In this case, the behavior of the system depends on the structure of the band-resolved Coulomb pseudopotential $\mu_{j j^{\prime}}^{*}$, which in turn is given by the chosen value of $c$. The simplest choice we can make is $c=0$, i.e. neglecting any repulsion between charge carriers pertaining to different

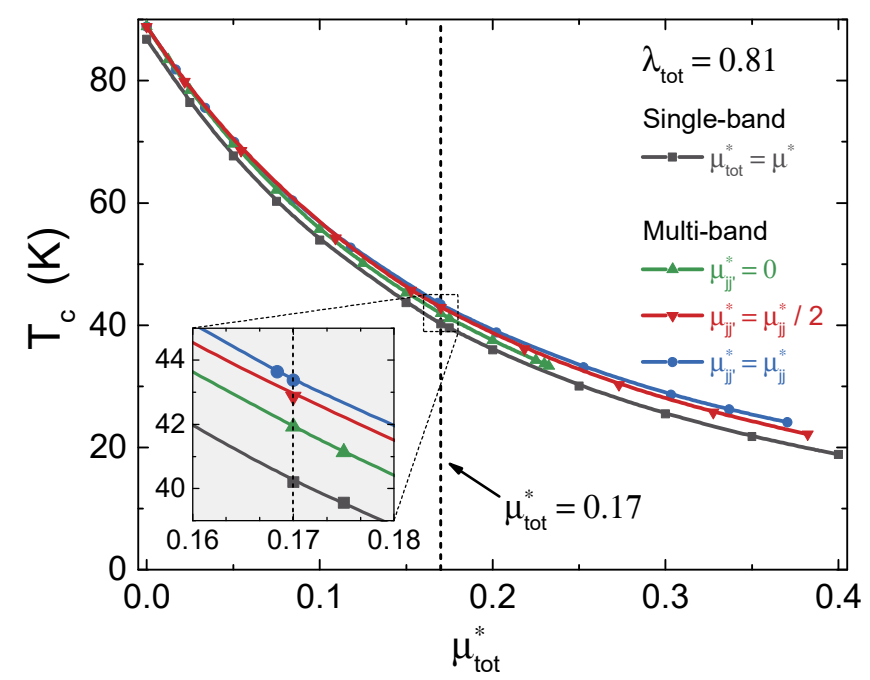

FIG. 3. Superconducting critical temperature $T_{\mathrm{c}}$ vs. the total effective Coulomb electron-electron interaction $\mu_{\text {tot }}^{*}$. The black line corresponds to the solution of the isotropic singleband $\left(j=j^{\prime}=1\right.$ and $\left.\mu^{*}=\mu_{\mathrm{tot}}^{*}\right) \mathrm{ME}$ equations (Eq. 15). The green, red and blue lines correspond to the solution of the isotropic multi-band $\left(j, j^{\prime}=1,2,3\right)$ Eliashberg equations with $c=0,1 / 2,1$ respectively for $\mu_{j j^{\prime}}^{*}$. 

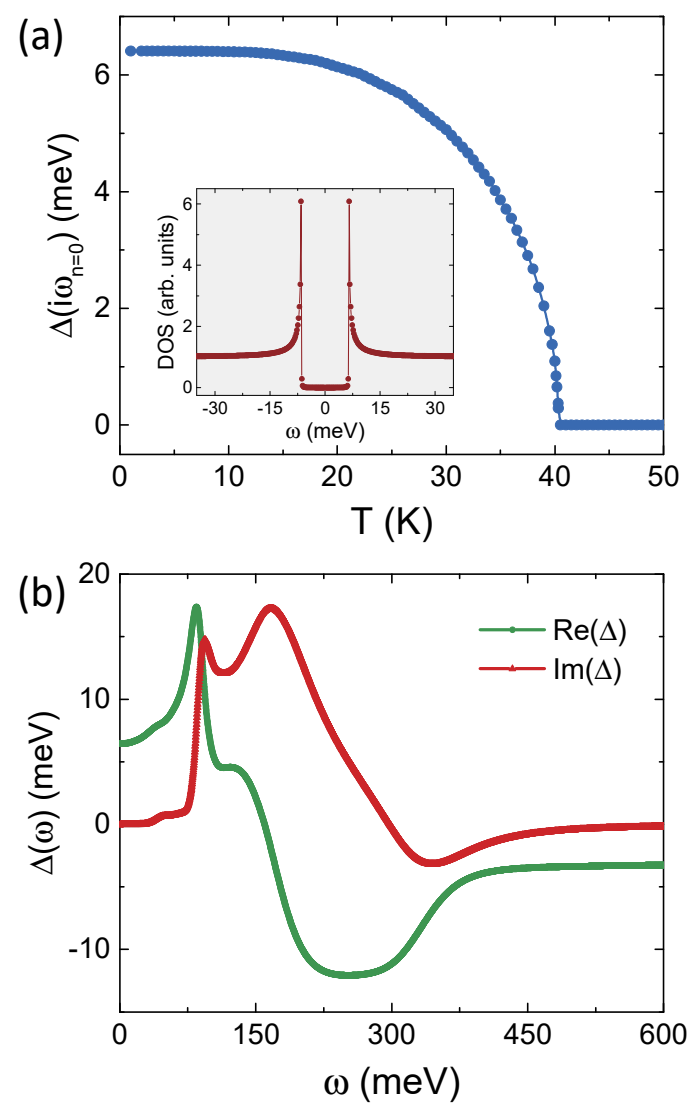

FIG. 4. (a) Superconducting order parameter on the imaginary-axis, $\Delta\left(i \omega_{n=0}\right)$, as a function of temperature $T$ obtained from the solution of the isotropic single-band MigdalEliashberg equations with $\mu^{*}=0.17$. The inset shows the single-band quasiparticle density of states (DOS) as a function of energy $\omega$; (b) Real (Re) and Imaginary (Im) part of the superconducting order parameter on the real-axis, $\Delta(\omega)$, obtained from the analytic continuation of $\Delta\left(i \omega_{n}\right)$ computed at $T=T_{c} / 10$.

bands (inter-band repulsion). We plot the corresponding dependence of $T_{\mathrm{c}}$ on $\mu_{\mathrm{tot}}^{*}$ as a solid green line in Fig. 3, and we find that the values of $T_{c}$ are slightly higher than those calculated in the single-band approximation. We also note that the solution of Eq. 15 becomes unstable for an equivalent $\mu_{\text {tot }}^{*} \gtrsim 0.23$, much larger than the physically-representative $\mu_{\mathrm{tot}}^{*}=0.17$ for which we find $T_{\mathrm{c}}=41.9 \mathrm{~K}$. Indeed, the most stable solution to the multi-band ME equations is found by setting $c=1 / 2$, as it can be extended up to $\mu_{\mathrm{tot}}^{*} \approx 0.38$. We plot the resulting dependence of $T_{\mathrm{c}}$ on $\mu_{\mathrm{tot}}^{*}$ as the solid red line in Fig. 3, from which we find a further increase in $T_{\mathrm{c}}$ with respect to both the single-band approximation and the multiband $c=0$ limit. For this intermediate strength of the inter-band repulsion, at the representative $\mu_{\text {tot }}^{*}=0.17$ we find $T_{\mathrm{c}}=42.9 \mathrm{~K}$. Finally, we consider the opposite limit $c=1$, where inter- and intra-band repulsions have exactly the same strength, and plot the corresponding $\mu_{\mathrm{tot}}^{*}$-dependence of $T_{\mathrm{c}}$ as as the solid blue line in Fig. 3.

\begin{tabular}{llllll}
\hline & & Single-band & \multicolumn{3}{c}{ Multi-band } \\
\hline & & & $c=0$ & $c=1 / 2$ & $c=1$ \\
\hline$T_{\mathrm{c}}$ & $\mathrm{K}$ & 40.3 & 41.9 & 42.9 & 43.4 \\
$\Delta$ & $\mathrm{meV}$ & 6.46 & $/$ & $/$ & $/$ \\
$\Delta_{1}$ & $\mathrm{meV}$ & $/$ & 7.11 & 7.43 & 7.59 \\
$\Delta_{2}$ & $\mathrm{meV}$ & $/$ & 6.50 & 6.47 & 6.46 \\
$\Delta_{3}$ & $\mathrm{meV}$ & $/$ & 5.74 & 5.54 & 5.44 \\
$r$ & & 3.72 & 3.62 & 3.60 & 3.58 \\
$r_{1}$ & & $/$ & 3.94 & 4.02 & 4.06 \\
$r_{2}$ & & $/$ & 3.60 & 3.50 & 3.45 \\
$r_{3}$ & & $/$ & 3.12 & 3.00 & 2.91 \\
\hline
\end{tabular}

TABLE III. Superconducting (SC) critical temperatures $T_{\mathrm{c}}$, SC order parameters for $T \rightarrow 0$ on the imaginary-axis $\Delta_{j}\left(i \omega_{n=0} ; T=0\right)$, and gap-to- $T_{\mathrm{c}}$ ratios $r_{j}$ (Eq. 19), as obtained from the solution of the isotropic ME equations with $\mu_{\mathrm{tot}}^{*}=0.17$. The multi-band cases are calculated using $c=$ $0, c=1 / 2$ and $c=1$ for $\mu_{j j^{\prime}}^{*}$. The indexes $j=1,2,3$ are labelled in Fig. 1(b).

For $\mu_{\mathrm{tot}}^{*} \lesssim 0.12$, this solution is basically equivalent to that at $c=1 / 2$. At larger values of $\mu_{\mathrm{tot}}^{*}, T_{\mathrm{c}}$ is found to slightly increase over the $T_{\mathrm{c}}$ calculated at $c=1 / 2$, although the solution to Eq. 15 becomes unstable at a slightly lower $\mu_{\text {tot }}^{*} \approx 0.37$. In this strong inter-band repulsion limit, we find $T_{\mathrm{c}}=43.4 \mathrm{~K}$ at the representative $\mu_{\mathrm{tot}}^{*}=0.17$. For all considered choices of $c$, the $T_{\mathrm{c}}$ calculated using the multi-band ME equations is larger than the one calculated using the single-band model: This indicates that the multi-band pairing strengthens the SC phase in the system, a behavior observed in several different superconductors ${ }^{67-70}$. In the case of fieldeffect-doped (111) diamond, our calculations show that the multi-band pairing enhances $T_{\mathrm{c}}$ between $\approx 4-8 \%$ at $\mu_{\mathrm{tot}}^{*}=0.17$ with respect to single-band paring, depending on the relative weight between intra-band and inter-band repulsions. More specifically, shifting the repulsion weight from intra-band terms $(c=0$ limit) to inter-band terms ( $c=1$ limit) is beneficial to $T_{\mathrm{c}}$, consistently with the largest element in the band-resolved e-ph coupling $\lambda_{j j^{\prime}}$ being the intra-band element $\lambda_{11}$ (which couples charge carriers within FS1).

We now consider the effect of multi-band pairing on the SC order parameter. In Fig. 5(a-c) we plot the three SC gaps $\Delta_{j}\left(i \omega_{n}\right)[j=1,2,3$, as labelled in Fig. 1(b)] computed by solving the isotropic multi-band ME equations along the imaginary-axis with $\mu_{\mathrm{tot}}^{*}=0.17$ and $c=0$ (a), $c=1 / 2(\mathrm{~b})$, and $c=1$ (c). The presence of multi-band pairing not only increases the $T_{\mathrm{c}}$, as we discussed previously, but also lifts the degeneracy between the three $\mathrm{SC}$ gaps as $T \rightarrow 0$. For any value of $c$, we find that $\Delta_{1}\left(i \omega_{n=0} ; T=0\right)$ is larger than the single-band $\Delta\left(i \omega_{n=0} ; T=0\right)$, and that $\Delta_{3}\left(i \omega_{n=0} ; T=0\right)$ is smaller. For $c=0, \Delta_{2}\left(i \omega_{n=0} ; T=0\right)$ is slighly larger than $\Delta\left(i \omega_{n=0} ; T=0\right)$, whereas for both $c=1 / 2$ and $c=1$ they are almost equal to each other. Overall, the splitting between the SC gaps increases as the weight of inter-band 


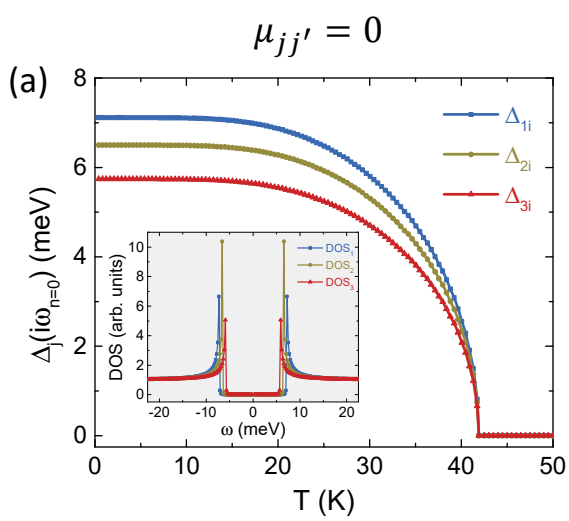

(d)

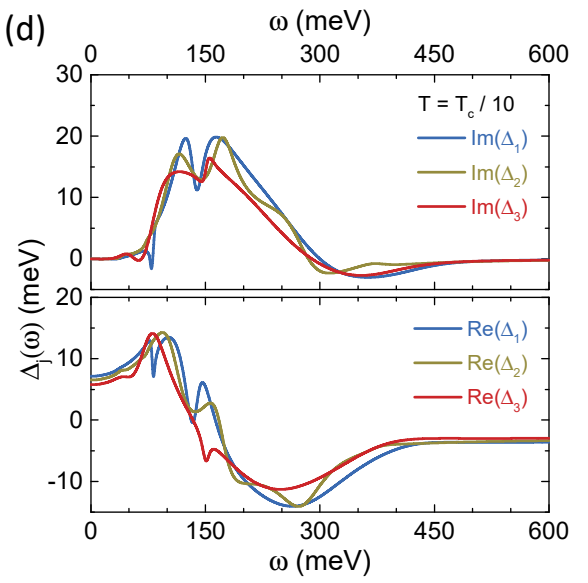

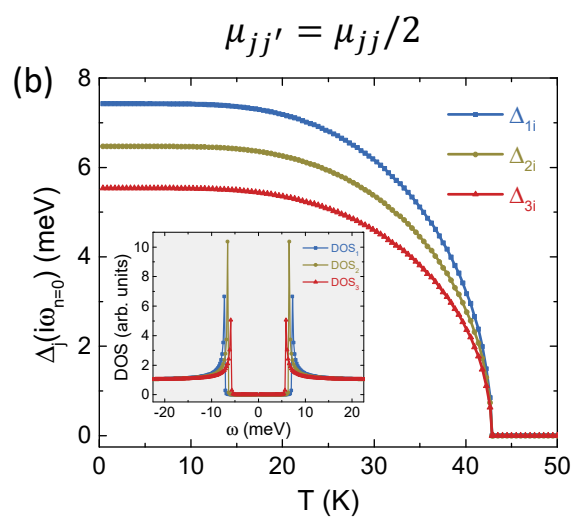

(e)

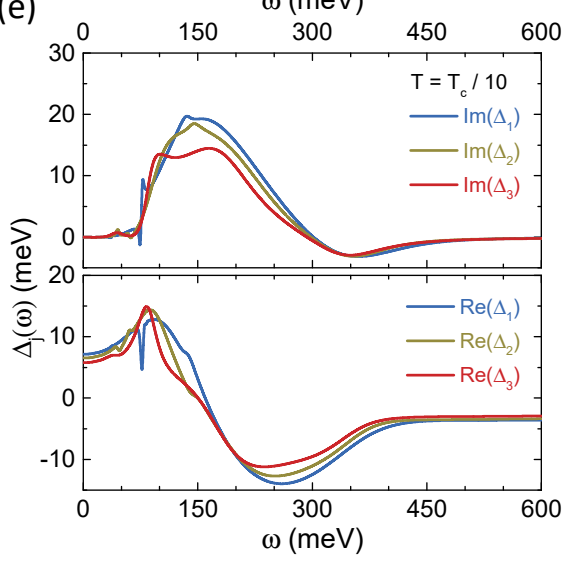

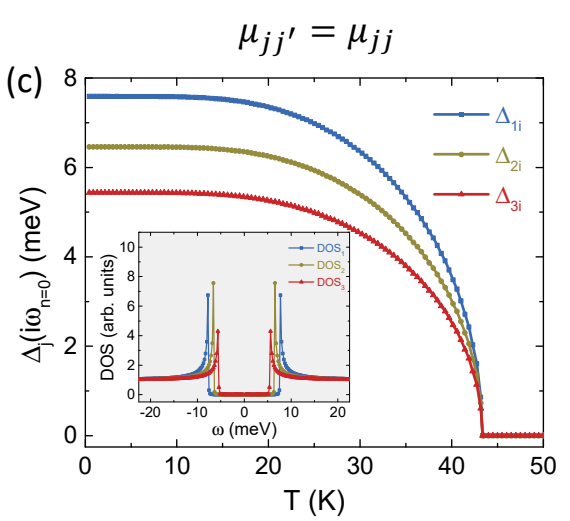

(f)

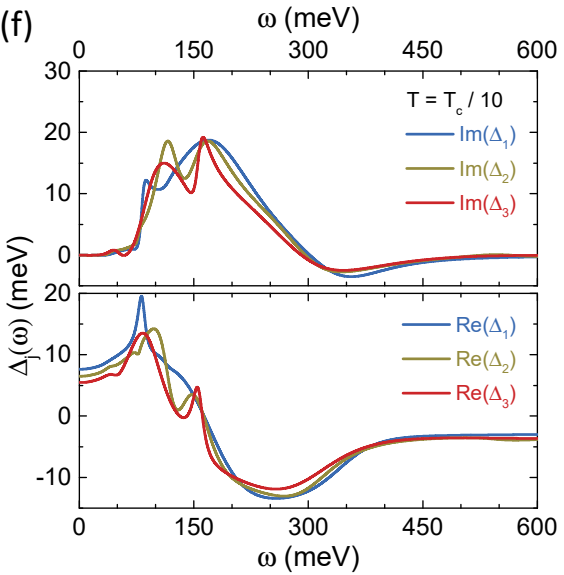

FIG. 5. (a-c) Band-resolved superconducting order parameters $\Delta_{j}\left(i \omega_{n=0}\right)[j=1,2,3$ as labelled in Fig. 1(b)] on the imaginaryaxis as a function of temperature $T$ obtained from the solution of the isotropic multi-band Migdal-Eliashberg equations at $\mu_{\mathrm{tot}}^{*}=0.17$ using (a) $c=0$, (b) $c=1 / 2$, and (c) $c=1$ for $\mu_{j j^{\prime}}^{*}$. In the inset we show the multi-band quasiparticle densities of states $\operatorname{DOS}_{j}$ as a function of energy $\omega$ calculated at $T=T_{\mathrm{c}} / 10$; (d-f) Real (Re) and Imaginary (Im) part of the superconducting order parameters $\Delta_{j}(\omega)$ on the real-axis obtained from the analytic continuation of $\Delta_{j}\left(i \omega_{n}\right)$ computed at $T=T_{\mathrm{c}} / 10$ using $\mu_{\text {tot }}^{*}=0.17$ and (d) $c=0,(\mathrm{e}) c=1 / 2$, and (f) $c=1$ for $\mu_{j j^{\prime}}^{*}$.

repulsion increases. For each SC gap, we also compute the corresponding gap-to- $T_{\mathrm{c}}$ ratio:

$$
r_{j}=\frac{2 \Delta_{j}\left(i \omega_{n=0} ; T=0\right)}{k_{B} T_{c}}
$$

from which we determine that in second band $r_{2}$ is close to the BCS value $r_{\mathrm{BCS}}=3.54$ for any value of $c$, whereas in the first and third bands $r_{1}$ and $r_{3}$ deviate almost symmetrically away from it, another typical behavior exhibited by multi-band superconductors ${ }^{71}$. Namely, $r_{1}>r_{\mathrm{BCS}}$ and increases at the increase of the inter-band repulsion weight, whereas $r_{3}<r_{\mathrm{BCS}}$ and decreases at the increase of the inter-band repulsion weight. The results of the multi-band model are consistent with those found single-band picture, as can be assessed by computing the weighted average of $r_{j}$ over the DOS per band $N_{\sigma, j}$ :

$$
r=\frac{\sum_{j} N_{\sigma, j}(0) r_{j}}{\sum_{j} N_{\sigma, j}(0)}
$$

We obtain $r=3.62$ for $c=0, r=3.60$ for $c=1 / 2$, and $r=3.58$ for $c=1$, close (even if smaller) to the single- band ratio $r=3.72$ we computed via Eq. 18, and decreasing at the increase of the inter-band repulsion weight. We summarize all the main parameters obtained by solving both the single- and multi-band isotropic ME equations in Table III.

In Fig. 5(d-f) we plot the analytic continuations on the real axis $\Delta_{j}(\omega)[j=1,2,3$ as labelled in Fig. 1(b)] of the SC order parameter computed on the imaginary axis at $T=T_{c} / 10$ and for the three different choices of inter-band repulsion weight: $c=0$ (d), $c=1 / 2$ (e), and $c=1$ (f). From these, we obtain the quasiparticle DOS for each band, which are plotted in the insets to Fig. $5(\mathrm{a}-\mathrm{c})$. Note that our choice to focus on $T=T_{c} / 10$ is not arbitrary: It is based on $T=T_{c} / 10$ being the standard condition for the reliable detection of multi-band features in the experimental tunnelling and Andreevreflection spectroscopy data ${ }^{73-76}$, and on the fact that computing the quasiparticle DOS at higher $T$ is beyond the validity of the analytic continuation and would thus require the solution of the much more computationally demanding $\mathrm{ME}$ equations on the real axis ${ }^{65,66}$. Once again, the presence of multi-band pairing is observed as 
a splitting of the single-peaked quasiparticle DOS computed in the single-band approximation into three different peaks, one for each SC gap. For all values of $c$, the quasiparticle DOS maintain the typical U-shape of s-wave superconductors, with larger inter-band repulsion weights leading to a more pronounced splitting between the peaks. From the experimental point of view, the detection of a multi-peaked quasiparticle spectrum would be a direct evidence for the multi-band nature of the pairing, and would allow to determine the actual value of the inter-band repulsion weight. This could be achieved by performing tunnelling spectroscopy measurements between buried boron-doped diamond electrodes electrically separated from the field-induced channel at the surface, similarly to the work described in Ref. 27.

Concerning the experimental feasibility of the proposed architecture, the most significant challenge lies in attaining the necessary $n_{d o p}=6 \times 10^{14} \mathrm{~cm}^{-2}$ to trigger the high-Tc SC state, as the current record for iongated hydrogenated intrinsic diamond is nearly one order of magnitude lower ${ }^{33}$ due to the small quantum capacitance of diamond ${ }^{30,36,37}$. Hole co-doping approaches have shown promise in this regard, combining ionic gating of hydrogenated surfaces with B substitution, and have allowed to reach $n_{\text {dop }} \sim 2 \times 10^{14} \mathrm{~cm}^{-230,36,37}$. Consequently, further improvements will be needed, such as developing ionic media capable of withstanding larger gate voltages, optimizing the $\mathrm{B}$ doping process to maximize the surface free carrier density, and increasing the quantum capacitance of the diamond surface by introducing an ultrathin spacer layer between diamond and the ionic gate $^{36,77}$.

\section{CONCLUSIONS}

In this work we have analyzed, by means of the isotropic Migdal-Eliashberg theory, the high- $\mathrm{T}_{\mathrm{c}}$ superconducting transition induced at the hydrogenated (111) surface of diamond when doped with holes via the electric field effect at a concentration of $n_{\text {dop }}=6 \times 10^{14} \mathrm{~cm}^{-2}$. Starting from the band-resolved electron-phonon spectral functions computed ab initio in a precedent work ${ }^{40}$, we first give a single-band description of the system and then we further extend the solution of the ME equations in order to account for the multi-band nature of the electronic dispersion relation at the Fermi level. The total effective electron-electron interaction $\mu_{t o t}^{*}$ is fixed by taking B-doped bulk diamond as reference, which gives $\mu^{*}=0.17$ according to the solution of the MigdalElishberg equations at a $\mathrm{B}$ doping concentration of $\sim$ $1.85 \%$ (i.e. $T_{\mathrm{c}} \approx 4 \mathrm{~K}$ ). In the single-band approximation we have that $\mu^{*}=\mu_{\text {tot }}^{*}$, while in the multi-band model we consider three possible Ansatz for the effectivecoulomb interaction matrix $\mu_{j j^{\prime}}^{*}$, always requiring that $\mu_{t o t}^{*}=\sum_{j j^{\prime}} N_{\sigma, j}(0) \mu_{j j^{\prime}}^{*} / \sum_{j} N_{\sigma, j}(0)$. The solution of the isotropic single-band $\mathrm{ME}$ equations shows that the $\mathrm{SC}$ phase transition is attained at $\sim 40 \mathrm{~K}$ with an s-wave gap value of $\sim 6.5 \mathrm{meV}$. However, when we take into account multi-band effects the $\mathrm{T}_{\mathrm{c}}$ is enhanced by $4-8 \%$, depending on the specific Ansatz for $\mu_{j j^{\prime}}^{*}$. For every choice of $\mu_{j j^{\prime}}^{*}$ we have analyzed, the band-resolved SC gaps $\Delta_{j}$ show an s-wave symmetry with $\Delta_{1}>\Delta_{2}>\Delta_{3}$. To offer a guideline for future experimental works aimed at determining the properties of the field-induced SC state, we also calculated a quantity sensitive to the multi-band pairing, namely the quasiparticle density of states, where we found a clear signature of multi-band effects. Finally, we would like to point out that - while we have so far discussed the most resonable choices for the inter- and intraband repulsion weights - more specific choices also allow to stabilize SC pairings not featuring the standard s-wave symmetry. For example, a choice of Coulomb pseudopotential such that $\mu_{j 3}^{*}=0.15$ and $\mu_{j k}^{*}=0$ leads to the stabilization of an $s \pm$ symmetry for the SC order parameter while keeping the source of pairing as purely e-ph driven.

\section{ACKNOWLEDGMENTS}

The authors acknowledge M. Calandra for fruitful scientific discussions. G.A.U. acknowledges support from the MEPhI Academic Excellence Project (Contract No. 02.a03.21.0005). E.P. acknowledges funding from the MIUR PRIN-2017 program (Grant No. 2017Z8TS5B - "Tuning and understanding Quantum phases in 2D materials - Quantum2D"). Computational resources were provided by hpc@polito (http://hpc.polito.it) and by CINECA, through the 'ISCRA C' project 'HP10C8P1FI'.
* davide.romanin@polito.it

† erik.piatti@polito.it

1 E. A. Ekimov, V. A. Sidorov, E. D. Bauer, N. N. Mel'nik, N. J. Curro, J. D. Thompson and S. M. Stishov. Superconductivity in diamond.. Nature 428, 542-545 (2004).

2 E. Bustarret. Superconducting diamond: an introduction. Phys. Status Solidi A 205, 997-1008 (2008).

${ }^{3}$ X. Blase, Ch. Adessi and D. Connetable. Role of the Dopant in the Superconductivity of Diamond. Phys. Rev.
Lett. 93, 237004 (2004).

${ }^{4}$ L. Boeri, J. Kortus and O. K. Andersen. Electronphonon superconductivity in hole-doped diamond: A firstprinciples study. J. Phys. Chem. Solids 67, 552-556 (2006).

5 F. Giustino, J. R. Yates, I. Souza, M. L. Cohen and S. G. Louie. Electron-Phonon Interaction via Electronic and Lattice Wannier Functions: Superconductivity in BoronDoped Diamond Reexamined. Phys. Rev. Lett. 98, 047005 (2007). 
6 J. E. Moussa and M. L. Cohen. Constraints on $T_{\mathrm{c}}$ for superconductivity in heavily boron-doped diamond. Phys. Rev. $B$ 77, 064518 (2008).

7 M. Calandra and F. Mauri. High- $T_{\mathrm{c}}$ Superconductivity in Superhard Diamondlike $\mathrm{BC}_{5}$. Phys. Rev. Lett. 101, 016401 (2008).

8 F. Caruso, M. Hoesch, P. Achatz, J. Serrano, M. Krisch, E. Bustarret and F. Giustino. Nonadiabatic Kohn Anomaly in Heavily Boron-Doped Diamond. Phys. Rev. Lett. 119, 017001 (2017).

9 Y.-H. Chen and C.-T. Hu. Defect structure and electron field-emission properties of boron-doped diamond films. Appl. Phys. Lett. 75, 2857 (1999).

10 V. L. Solozhenko, O. O. Kurakevych, D. Andrault, Y. Le Godec and M. Mezouar. Ultimate Metastable Solubility of Boron in Diamond: Synthesis of Superhard Diamondlike $\mathrm{BC}_{5}$. Phys. Rev. Lett. 102, 015506 (2009).

11 M. I. Landstrass and K. V. Ravi. Resistivity of chemical vapor deposited diamond films. Appl. Phys. Lett. 55, 975 (1989).

12 F. Maier, M. Riedel, B. Mantel, J. Ristein, and L. Ley. Origin of Surface Conductivity in Diamond. Phys. Rev. Lett. 85, 3472 (2000).

13 P. Strobel, M. Riedel, J. Ristein, and L. Ley. Surface transfer doping of diamond. Nature 430, 439 (2004).

14 H. Kawarada. Hydrogen-terminated diamond surfaces and interfaces. Surf. Sci. Rep. 26, 205 (1996).

15 C. E. Nebel, F. Ertl, C. Sauerer, M. Stutzmann, C. F. O. Graeff, P. Bergonzo, O. A. Williams, and R. B. Jackman. Low temperature properties of the p-type surface conductivity of diamond. Diamond Relat. Mater. 11, 351 (2002).

16 C. E. Nebel, B. Rezek, and A. Zrenner. Electronic properties of the 2D-hole accumulation layer on hydrogen terminated diamond. Diamond Relat. Mater. 13, 2031 (2004).

17 M. T. Edmonds, C. I. Pakes, and L. Ley. Self-consistent solution of the Schrödinger-Poisson equations for hydrogenterminated diamond. Phys. Rev. B 81, 085314 (2010).

18 M. T. Edmonds, L. H. Willems van Beveren, O. Klochan, J. Cervenka, K. Ganesan, S. Prawer, L. Ley, A. R. Hamilton, and C. I. Pakes. Spin-Orbit Interaction in a TwoDimensional Hole Gas at the Surface of Hydrogenated Diamond. Nano Lett. 15, 16 (2015).

19 D. Daghero, F. Paolucci, A. Sola, M. Tortello, G. A. Ummarino, M. Agosto, R. S. Gonnelli, J. R. Nair and C. Gerbaldi. Large Conductance Modulation of Gold Thin Films by Huge Charge Injection via Electrochemical Gating. Phys. Rev. Lett. 108, 066807 (2012).

${ }^{20}$ K. Ueno, H. Shimotani, H. Yuan, J. Ye, M. Kawasaki, and I. Iwasa. Field-Induced Superconductivity in Electric Double Layer Transistors. J. Phys. Soc. Jpn. 83, 032001 (2014).

21 E. Piatti, A. Sola, D. Daghero, G. A. Ummarino, F. Laviano, J. R. Nair, C. Gerbaldi, R. Cristiano, A. Casaburi, and R. S. Gonnelli. Superconducting transition temperature modulation in $\mathrm{NbN}$ via EDL gating. J. Supercond. Novel Magn. 29, 587-591 (2016).

${ }^{22}$ L. J. Li, E. C. T. O'Farrel, K. P. Loh, G. Eda, B. Özyilmaz, and A. H. Castro Neto. Controlling many-body states by the electric-field effect in a two-dimensional material. $\mathrm{Na}$ ture 529, 185 (2016).

23 E. Piatti, D. Daghero, G. A. Ummarino, F. Laviano, J. R. Nair, R. Cristiano, A. Casaburi, C. Portesi, A. Sola and R. S. Gonnelli. Control of bulk superconductivity in a BCS superconductor by surface charge doping via electrochemical gating. Phys. Rev. B 95, 140501 (2017).

${ }^{24} \mathrm{X}$. Xi, H. Berger, L. Forró, J. Shan, and K. F. Mak. Gate Tuning of Electronic Phase Transitions in TwoDimensional $\mathrm{NbSe}_{2}$. Phys. Rev. Lett. 117, 106801 (2016).

${ }^{25}$ R. S. Gonnelli, E. Piatti, A. Sola, M. Tortello, F. Dolcini, S. Galasso, J. R. Nair, C. Gerbaldi, E. Cappelluti, M. Bruna, and A. C. Ferrari. Weak localization in electric-double-layer gated few-layer graphene. $2 D$ Mater. 4, 035006 (2017).

26 E. Piatti, Q. H. Chen, and J. T. Ye. Strong dopant dependence of electric transport ion-gated $\mathrm{MoS}_{2}$. Appl. Phys. Lett. 111, 013106 (2017).

27 D. Costanzo, H. Zhang, B. A. Reddy, H. Berger, and A. F. Morpurgo. Tunnelling spectroscopy of gate-induced superconductivity in $\mathrm{MoS}_{2}$. Nat. Nanotechnol. 13, 483-488 (2018).

28 E. Piatti, Q. H. Chen, M. Tortello, J. T. Ye, and R. S. Gonnelli. Possible charge-density-wave signatures in the anomalous resistivity of Li-intercalated multilayer $\mathrm{MoS}_{2}$. Appl. Surf. Sci. 461, 269-275 (2018).

29 E. Piatti, T. Hatano, D. Daghero, F. Galanti, C. Gerbaldi, S. Guastella, C. Portesi, I. Nakamura, R. Fujimoto, K. Iida, H. Ikuta, and R. S. Gonnelli. Ambipolar suppression of superconductivity by ionic gating in optimallydoped $\mathrm{BaFe}_{2}(\mathrm{As}, \mathrm{P})_{2}$ ultrathin films. Phys. Rev. Materials 3, 044801 (2019).

30 E. Piatti, D. Romanin, D. Daghero and R. S. Gonnelli. Two-dimensional hole transport in ion-gated diamond surfaces: A brief review. Low Temp. Phys. 45(11), 1143-1155 (2019).

31 T. Yamaguchi, E. Watanabe, H. Osato, D. Tsuya, K. Deguchi, T. Watanabe, H. Takeya, Y. Takano, S. Kurihara, and H. Kawarada. Low-Temperature Transport Properties of Holes Introduced by Ionic Liquid Gating in HydrogenTerminated Diamond Surfaces. J. Phys. Soc. Jpn. 82, 074718 (2013).

32 Y. Takahide, H. Okazaki, K. Deguchi, S. Uji, H. Takeya, Y. Takano, H. Tsuboi, and H. Kawarada. Quantum oscillations of the two-dimensional hole gas at atomically flat diamond surfaces. Phys. Rev. B 89, 235304 (2014).

33 G. Akhgar, O. Klochan, L. H. Willems van Beveren, M. T. Edmonds, F. Maier, B. J. Spencer, J. C. McCallum, L. Ley, A. R. Hamilton, and C. I. Pakes. Strong and Tunable Spin-Orbit Coupling in a Two-Dimensional Hole Gas in Ionic-Liquid Gated Diamond Devices. Nano Lett. 16, 3768 (2016).

34 Y. Takahide, Y. Sasama, M. Tanaka, H. Takeya, Y. Takano, T. Kageura, and H. Kawarada. Spininduced anomalous magnetoresistance at the (100) surface of hydrogen-terminated diamond. Phys. Rev. B 94, 161301(R) (2016).

35 G. Akhgar, L. Ley, D. L. Creedon, A. Stacey, J. C. McCallum, A. R. Hamilton, and C. I Pakes. $g$-factor and wellwidth fluctuations vs carrier density in the $2 \mathrm{~d}$ hole accumulation layer of transfer-doped diamond. Phys. Rev. B 99, 035159 (2019).

36 E. Piatti, A. Pasquarelli, and R. S. Gonnelli. Orientationdependent electric transport and band filling in hole codoped epitaxial diamond films. Appl. Surf. Sci., 528, 146795 (2020).

37 E. Piatti, F. Galanti, G. Pippione, A. Pasquarelli, and R. S. Gonnelli. Towards the insulator-to-metal transition in iongated nanocrystalline diamond films. Eur. Phys. J. Spec. 
Top. 228, 689 (2019).

38 K. Nakamura, S. H. Rhim, A. Sugiyama, K. Sano, T. Akiyama, T. Ito, M. Weinert, and A. J. Freeman. Electricfield-driven hole carriers and superconductivity in diamond. Phys. Rev. B 87, 214506 (2013).

${ }^{39}$ K. Sano, T. Hattori, and K. Nakamura. Role of surfacebound hole states in electric-field-driven superconductivity at the (110)-surface of diamond. Phys. Rev. B 96, 155144 (2017).

40 D. Romanin, Th. Sohier, D. Daghero, F. Mauri, R. S. Gonnelli and M. Calandra. Electric field exfoliation and high- $T_{\mathrm{c}}$ superconductivity in field-effect hole-doped hydrogenated diamond (111). Appl. Surf. Sci. 496, 143709 (2019).

41 W. L. McMillan. Transition Temperature of StrongCoupled Superconductors. Phys. Rev. 167, 331 (1968).

42 P. B. Allen, R. C. Dynes. Transition temperature of strongcoupled superconductors reanalyzed. Phys. Rev. B 12, 905 (1975).

43 G. M. Eliashberg. Interactions between electrons and lattice vibrations in a superconductor. Sov. Phys. JETP 11, 696 (1960).

44 G. M. Eliashberg. Interactions between electrons and lattice vibrations in a superconductor. Zh. Eksp. Teor. Fiz. 38, 966 (1960).

45 P.Giannozzi et al. QUANTUM ESPRESSO: a modular and open-source software project for quantum simulations of materials. J. Phys. Condens. Matter 21, 395502 (2009).

46 P.Giannozzi et al. Advanced capabilities for materials modelling with Quantum ESPRESSO. J. Phys. Condens. Matter 29, 465901 (2017).

47 T. Sohier, M.Calandra, F. Mauri. Density functional perturbation theory for gated two-dimensional heterostructures: Theoretical developments and application to flexural phonons in graphene. Phys. Rev. B 96, 075448 (2017).

48 T. Brumme, M. Calandra, and F. Mauri. Electrochemical doping of few-layer $\mathrm{ZrNCl}$ from first principles: Electronic and structural properties in field-effect configuration. Phys. Rev. B 89, 245406 (2014).

49 T. Sohier, D. Campi, N. Marzari, and M. Gibertini. Mobility of two-dimensional materials from first principles in an accurate and automated framework. Phys. Rev. Materials 2, 114010 (2018).

50 T. Brumme, M. Calandra, and F. Mauri. First-principles theory of field-effect doping in transition-metal dichalcogenides: Structural properties, electronic structure, Hall coefficient, and electrical conductivity. Phys. Rev. B 96, 075448 (2017).

${ }^{51}$ T. Brumme, M. Calandra, and F. Mauri. Determination of scattering time and of valley occupation in transitionmetal dichalcogenides doped by field effect. Phys. Rev. B 96, 075448 (2017).

${ }^{52}$ E. Piatti, D. Romanin, and R. S. Gonnelli. Mapping multivalley Lifshitz transitions induced by field-effect doping in strained $\mathrm{MoS}_{2}$ nanolayers. J. Phys. Condens. Matter 31, 114002 (2019).

53 D. Romanin, T. Brumme, D. Daghero, R. S. Gonnelli, and E. Piatti. Strong band-filling-dependence of the scattering lifetime in gated $\mathrm{MoS}_{2}$ nanolayers induced by the opening of intervalley scattering channels. J. Appl. Phys. 128, 063907 (2020).

${ }^{54}$ E. Piatti, D. Romanin, R. S. Gonnelli, and D. Daghero. Anomalous screening of an electrostatic field at the surface of niobium nitride. Appl. Surf. Sci. 461, 17-22 (2018).
55 D. Romanin. Field-effect induced superconductivity in surfaces of tetrahedrally coordinated semiconductors: the case of (111) hydrogenated silicon. arXiv:2001.08952.

56 A. A. Mostofi, J. R. Yates, G. Pizzi, Y. S. Lee, I. Souza, D. Vanderbilt, and N. Marzari. An updated version of wannier90: A tool for obtaining maximally-localised Wannier functions. Comput. Phys. Commun. 185, 2309 (2014).

57 M. Calandra, G. Profeta, F. Mauri. Adiabatic and nonadiabatic phonon dispersion in a Wannier function approach. Phys. Rev. B 82, 165111 (2010).

58 A. Sanna, S. Pittalis, J. K. Dewhurst, M. Monni, S. Sharma, G. Ummarino, S. Massidda, and E. K. U. Gross. Phononic self-energy effects and superconductivity in $\mathrm{CaC}_{6}$. Phys. Rev. B 85, 184514 (2012).

59 P. Morel, P. W. Anderson. Calculation of the Superconducting State Parameters with Retarded Electron-Phonon Interaction. Phys. Rev. 125, 1263 (1962).

60 P. B. Allen and B. Mitrovic, in Solid State Physics edited by H. Ehrenreich, F. Seitz and D. Turnbull (Academic, New York), Vol. 37, p.1 (1982).

61 J. P. Carbotte. Properties of boson-exchange superconductors. Rev. Mod. Phys. 62, 1027 (1990).

${ }^{62}$ G. A. Ummarino, Eliashberg Theory. In: Emergent Phenomena in Correlated Matter, edited by E. Pavarini, E. Koch, and U. Schollwöck, Forschungszentrum Jülich $\mathrm{GmbH}$ and Institute for Advanced Simulations, pp.13.113.36 (2013) ISBN 978-3-89336-884-6.

63 G. A. Ummarino. Phenomenology of $\mathrm{CaKFe}_{4} \mathrm{As}_{4}$ explained in the framework of four bands Eliashberg theory. Physica $C$ 529, 50 (2016).

${ }^{64}$ G. A. Baker Jr., Essentials of Padé Approximants, Acaemic Press, New York (1975).

${ }^{65}$ H. J. Videberg and J. W. Serene. Solving the Eliashberg equations by means of N-point Padé approximants. J. Low Temp. Phys. 29, 179 (1977).

66 C. R. Leavens and D. S. Ritchie. Extension of the N-point Padé approximants solution of the Eliashberg equations to $T \sim T_{\mathrm{c}}$. Solid State Commun. 53, 137-142 (1985).

67 G. A. Ummarino, R. S. Gonnelli, S. Massidda and A. Bianconi. Two-band Eliashberg equations and the experimental $T_{\mathrm{c}}$ of the diboride $\mathrm{Mg}_{1-\mathrm{x}} \mathrm{Al}_{\mathrm{x}} \mathrm{B}_{2}$. Physica $C$ 407, 121 (2004).

68 W. E. Pickett. Superconductivity: 2D Physics, Unknown Mechanisms, Current Puzzles. In: Emergent Phenomena in Correlated Matter. (Forschungszentrum Jülich GmbH and Institute for Advanced Simulations: Jülich, Germany, 2013).

69 R. S. Gonnelli, D. Daghero, M. Tortello, G. A. Ummarino, Z. Bukowski, J. Karpinski, P. G. Reuvekamp, R. K. Kremer, G. Profeta, K. Suzuki, and K. Kuroki, FermiSurface topological phase transition and horizontal OrderParameter nodes in $\mathrm{CaFe}_{2} \mathrm{As}_{2}$ under pressure. Sci. Rep. 6, 26394 (2016)

70 E. Piatti, D. De Fazio, D. Daghero, S. R. Tamalampudi, D. Yoon, A. C. Ferrari, and R. S. Gonnelli. Multi-Valley Superconductivity in Ion-Gated $\mathrm{MoS}_{2}$ Layers. Nano Lett. 18, 4821-4830 (2018).

71 D. S. Inosov, J. T. Park, A. Charnukha, Yuan Li, A. V. Boris, B. Keimer, and V. Hinkov. Crossover from weak to strong pairing in unconventional superconductors. Phys. Rev. B 83, 214520 (2011).

72 G. A. Ummarino, D. Daghero, M. Tortello, and R. S. Gonnelli. Predictions of Multiband $s \pm$ Strong-Coupling Eliashberg Theory Compared to Experimental Data in 
Iron Pnictides. J. Supercond. Novel Magn. 24, 247-253 (2011).

73 D. Daghero and R. S. Gonnelli. Probing multiband superconductivity by point-contact spectroscopy. Supercond. Sci. Technol. 23, 043001 (2010).

${ }^{74}$ R. S. Gonnelli, D. Daghero, G. A. Ummarino, V. A. Stepanov, J. Jun, S. M. Kazakov, and J. Karpinski. Direct Evidence for Two-Band Superconductivity in $\mathrm{MgB}_{2}$ Single Crystals from Directional Point-Contact Spectroscopy in Magnetic Fields. Phys. Rev. Lett. 89, 247004 (2002).

75 R. S. Gonnelli, D. Daghero, D. Delaude, M. Tortello, G. A. Ummarino, V. A. Stepanov, J. S. Kim, R. K. Kremer,
A. Sanna, G. Profeta, and S. Massidda. Evidence for Gap Anisotropy in $\mathrm{CaC}_{6}$ from Directional Point-Contact Spectroscopy. Phys. Rev. Lett. 100, 207004 (2008).

76 D. Daghero, M. Tortello, G. A. Ummarino, E. Piatti, G. Ghigo, T. Hatano, T. Kawaguchi, H. Ikuta, and R. S. Gonnelli. Decoupling of critical temperature and superconducting gaps in irradiated films of a Fe-based superconductor. Supercond. Sci. Technol. 31, 034005 (2018).

77 H. Zhang, C. Berthod, H. Berger, T. Giamarchi, and A. F. Morpurgo. Band Filling and Cross Quantum Capacitance in Ion-Gated Semiconducting Transition Metal Dichalcogenide Monolayers. Nano Lett. 19, 8836-8845 (2019). 